Artículo

\title{
Estimación de la rentabilidad agrícola de las empresas rurales de la mixteca Oaxaqueña
}

\author{
Gerónimo Barrios Puente ${ }^{1 \S}$ \\ Arisbeth Rosales Hortiales ${ }^{1}$ \\ Dora María Sangerman-Jarquín ${ }^{2}$ \\ Francisco Pérez Soto ${ }^{1}$ \\ Mariano González López ${ }^{1}$ \\ Luis Reyes Muro ${ }^{3}$ \\ ${ }^{1}$ Universidad Autónoma Chapingo. Carretera México-Texcoco km 38.5, Chapingo, Texcoco, Estado de \\ México. CP. 56230. Tel. 595 9521500. (arisroho@hotmail.com; perezsotofco@gmail.com; \\ marianohuanda81@gmail.com). ${ }^{2}$ Campo Experimental Valle de México-INIFAP. Carretera Los Reyes- \\ Texcoco km 13.5, Coatlinchán, Texcoco, Estado de México. CP. 56250. (sangerman.dora@inifap.gob.mx). \\ ${ }^{3}$ Campo Experimental Pabellón-INIFAP. Carretera Aguascalientes-Zacatecas km 32.5, Pabellón de \\ Arteaga, Aguascalientes. CP. 20660. Tel. 800088 2222, ext. 82501. (reyes.luis@inifap.gob.mx). \\ §Autor para correspondencia: gbarriospuente55@gmail.com.
}

\section{Resumen}

En la región Mixteca en Oaxaca predomina la pobreza rural y la actividad económica común es la agricultura; sin embargo, el sector agrícola es precario y no ha reducido la pobreza. Existen pequeñas parcelas, productos tradicionales de poco valor agregado, técnicas antiguas en tecnología y no hay inversión en capital. En este sentido es necesario conocer los beneficios económicos que generan las unidades productivas (UP), visualizándolas como empresas rurales. El objetivo de la investigación fue hacer una aproximación a la estimación de la rentabilidad de la producción agrícola de las empresas rurales de la región Mixteca de Oaxaca para el año 2007 y conocer cuáles fueron los principales factores que influyeron en dicha rentabilidad, para ello se estimaron los ingresos y costos de las empresas rurales por municipio cuya diferencia arrojó el valor de rentabilidad, y mediante mínimos cuadrados ordinarios se determinaron los factores que influyen en ella. Los resultados indicaron que la rentabilidad por empresas rurales estuvo en el rango de \$19000 a $\$ 13000$, por municipios de \$-4 millones a \$4 millones y por superficie de \$-9 000 a \$6 500 por hectárea. La producción, semillas mejoradas y tamaño de UP influyeron positivamente en el valor de rentabilidad, mientras que el autoconsumo, carecer de infraestructura, la propiedad comunal e ingresos derivados de apoyos gubernamentales la disminuyen. Finalmente, es necesario fomentar la asociación de productores que permita incrementar la producción y aumentar las prácticas de comercialización para generar mayores beneficios derivados de la agricultura.

Palabras clave: agricultura, autoconsumo, ganancias, unidades productivas.

Recibido: mayo de 2021

Aceptado: agosto de 2021 


\section{Introducción}

El sector rural es, desde hace ya varias décadas, el sector social y productivo con mayores condiciones de rezago y vulnerabilidad en el país. De acuerdo con CONEVAL (2019) a nivel nacional $41.9 \%$ de la población se encuentra en situación de pobreza (equivalente a 52 millones de personas) y $7.4 \%$ se ubica en pobreza extrema ( 9 millones de personas), la mayor parte de la pobreza se ubica en los estados del sureste, siendo Chiapas con $76 \%$ de su población en pobreza la entidad federativa con el mayor porcentaje, seguido de Guerrero con 66.5\% y Oaxaca con 66.4\%; es decir, en general la pobreza se ubica en los estados que tienen el mayor porcentaje de población rural, presentan marginación, escases de inversión pública y falta de acceso a mercados (Ortiz y Ríos, 2013). Particularmente, Oaxaca es la entidad donde $70 \%$ de sus localidades tienen menos de 2500 habitantes y ocupa el último lugar en los índices de competitividad estatal según el Gobierno del Estado de Oaxaca (2016), esta situación influye en los elevados índices de marginación y pobreza característicos del estado.

Al interior de la entidad, la región mixteca ubicada al noroeste del estado alberga el 11.8\% de la población estatal, $77.4 \%$ son comunidades rurales y $73 \%$ de la población se encuentra en situación de pobreza, únicamente $35 \%$ de la población es económicamente activa, siendo los servicios la mayor ocupación (35\%), seguida por actividades agropecuarias (26\%) y 32\% de los ingresos son de hasta 1 salario mínimo (Universidad Tecnológica de la Mixteca, 2017).

Dado que tres cuartas partes de la población mixteca es rural y la actividad agricultura sigue siendo la fuente de ingresos de un importante número de personas es transcendental conocer su economía agrícola, pero analizarla con una visión empresarial, ya que las UP agrícolas también son empresas en el sentido que los agricultores producen bienes (productos principalmente alimenticios) de los que buscan obtener una rentabilidad positiva, que disponen de tecnología, organización y generan empleos (Parra, 2000).

De acuerdo con Mallo et al. (2000), la producción integra elementos técnicos y económicos, en lo técnico implica la transformación de unos bienes en otros que, para el caso del sector agrícola implica un proceso biológico y en lo económico este proceso implica un valor superior al de los medios utilizados en su obtención. La producción agrícola se realiza en las denominadas UP y depende de factores como superficie del terreno, mano de obra empleada, capital invertido y valor de los beneficios anuales (Molina de Paredes, 2017).

La presente investigación tuvo como objetivo hacer una aproximación a la estimación de la rentabilidad de la producción agrícola en las UP (empresas rurales) de la región mixteca de Oaxaca para el año 2007 y conocer cuáles fueron los principales factores que influyeron en dicha rentabilidad, con el fin de establecer una línea base para posteriores investigaciones y hacer recomendaciones en beneficio de la economía agrícola de la región. Este estudio también permitirá encontrar parámetros de utilidad para una siguiente etapa de investigación.

La hipótesis planteada considerando estudios similares (Espinosa et al., 2013), es que las empresas rurales de la mixteca oaxaqueña en el año 2007 tuvieron rentabilidades promedio menores a $\$ 15$ 000 pesos anuales y en algunos casos rentabilidades negativas, los factores que influyeron significativamente fueron la mano de obra, la fertilización y la superficie cosechada (Molina de Paredes, 2017). 


\section{Materiales y métodos}

La investigación consistió en hacer una aproximación cuantitativa a la estimación de la rentabilidad monetaria de las empresas rurales de la región mixteca de Oaxaca para el año 2007, año elegido debido a que los principales datos a nivel municipal necesarios para la investigación se encuentran únicamente en el Censo Nacional Agrícola, Ganadero y Forestal 2007, censo agrícola más reciente realizado en México, el cual, por el nivel de análisis no es sustituido por la reciente Encuesta Nacional Agropecuaria 2017 (Centro de Estudios para el Desarrollo Rural Sustentable y la Soberanía Alimentaria, 2019).

La información se obtuvo de bases de datos oficiales publicados por instancias como el Instituto Nacional de Estadística y Geografía (INEGI), Censo Nacional Agrícola, Ganadero y Forestal 2007 (CNAGF), Servicio de Información Agroalimentaria y Pesquera (SIAP), Consejo Nacional de Evaluación de la Política de Desarrollo Social (CONEVAL) y el Instituto Nacional de Investigaciones Forestales, Agrícolas y Pecuarias (INIFAP). El universo de estudio fueron los municipios rurales de la región mixteca de Oaxaca, tomando en cuenta el número de UP existentes en cada municipio.

El número de municipios rurales fueron 151 del total de 155 municipios el criterio usado para determinar las poblaciones rurales fue basado en Unikel et al. (1976) que propone que una población rural tiene menos de 15000 habitantes. Para los 151 municipios rurales de la región mixteca, se obtuvieron datos para el año 2007 de las siguientes variables: 1)Variables sociales: población, nivel de escolaridad y niveles de pobreza; 2)variables económicas: población económicamente activa, mano de obra remunerada y no remunerada, valor de la producción, actividades económicas, ingresos familiares, y costos de producción; y 3)variables productivas: número de unidades de producción, superficie agrícola, superficie de riego y temporal, uso de maquinaria, uso de fertilizantes, uso de semillas mejoradas, autoconsumo, comercialización, y volumen de producción. El referente para la información de costos de producción fue la Agenda Técnica Agrícola de Oaxaca (2015), publicada por SAGARPA, SENASICA e INIFAP.

\section{Estimación de rentabilidad}

Para la determinación de la rentabilidad se basó en Ayala et al (2013). Donde: rentabilidad $=$ ingresos totale - costos totales. Dada la escasa disponibilidad de datos sobre costos (el censo no brinda información de costos de producción específicos para cada UP ni para cada municipio), el cálculo de los ingresos y costos se hizo con el diseño del siguiente procedimiento: Donde: IDAM= ingreso de la agricultura en el municipio (pesos); i: cultivo (café, frijol, maíz, trigo); $\mathrm{S}=$ superficie cultivada en el municipio (ha); $\mathrm{R}=$ rendimiento $\left(\mathrm{t} \mathrm{ha}^{-1}\right) ; \mathrm{P}=$ precio (pesos); IDEA= ingreso de la empresa agrícola (pesos); $\mathrm{E}=$ número de empresas en el municipio; $\mathrm{IDAH}=$ ingreso promedio de la agricultura por hectárea (pesos).

Ingresos: IDAM $=\sum_{\mathrm{i}=1}^{4} \mathrm{~S}_{\mathrm{i}} \mathrm{R}_{\mathrm{i}} \mathrm{P}_{\mathrm{i}} ;$ IDEA $=\frac{\text { IDAM }}{\mathrm{E}} ; \mathrm{IDAH}=\frac{\text { IDAM }}{\mathrm{S}}$

Costos:

$\mathrm{CADAM}=\mathrm{TATAM}^{*} \mathrm{~W} * \mathrm{t}+\sum_{\mathrm{i}=1}^{4}\left(\mathrm{~S}_{\mathrm{i}} \mathrm{CFH}_{\mathrm{i}} \% \mathrm{FS}_{\mathrm{i}}\right.$ proFH $_{\mathrm{i}}+\mathrm{S}_{\mathrm{i}} \mathrm{CMH}_{\mathrm{i}} \% \mathrm{MS}_{\mathrm{i}}$ proMH $\left._{\mathrm{i}}+\mathrm{S}_{\mathrm{i}} \mathrm{CSH}_{\mathrm{i}} \% \mathrm{SS}_{\mathrm{i}} \mathrm{proSH}_{\mathrm{i}}\right)$ ; $\mathrm{CADEA}=\frac{\mathrm{CADAM}}{\mathrm{E}} ; \mathrm{CADAH}=\frac{\mathrm{CADAM}}{\mathrm{S}}$. 
Donde:

CADAM: costo agregado de la agricultura en el municipio (pesos)

t: días trabajados

proFH: proporción del fertilizante usado con respecto al recomendado por hectárea

proMH: proporción del uso de maquinaria respecto al recomendado por hectárea

proSH: proporción del uso de semilla mejorada respecto al recomendado por hectárea.
TATAM: trabajo agrícola $\mathrm{W}$ : salario total acumulado en el municipio

CFH: costo del fertilizante por hectárea (pesos)

CMH: costo del uso de la maquinaria por hectárea (pesos)

CSH: costo de la semilla mejorada por hectárea (pesos)

CADEA: costo agregado de la empresa agrícola (pesos)
$\%$ FS: porcentaje de la superficie que es fertilizada

\%MS: porcentaje de la superficie que usa maquinaria

$\%$ SS: porcentaje de la superficie que usa semilla mejorada

CADAH: costo agregado de la agricultura por hectárea (pesos)

Rentabilidad:(ganancia): GADAM=IDAM-CADAM; GADEA=IDEA-CADEA; GADAH=IDAH-CADAH.

Donde: GADAH= ganancia de la agricultura por hectárea (pesos); CADEA= costo agregado de la empresa agrícola (pesos); CADAH= costo agregado de la agricultura por hectárea (pesos).

\section{Factores que influyen en la rentabilidad}

La relación entre diversas variables sociales, económicas y productivas investigadas (variables explicativas) con el nivel de rentabilidad obtenido de las UP de los municipios (variable dependiente) calculado previamente fue analizada mediante el modelo de regresión lineal múltiple y MCO, donde se obtuvo una ecuación del tipo $\mathrm{Y}=\beta_{0}+\beta_{1} \mathrm{x}_{1}+\beta_{\mathrm{n}} \mathrm{x}_{\mathrm{n}}+\mathrm{e}$, los coeficientes o parámetros $\beta_{1}, \beta_{2}, \beta_{\mathrm{n}}$ denotan la magnitud del efecto que las variables explicativas $\mathrm{x}_{1}, \mathrm{x}_{2}, \mathrm{x}_{\mathrm{n}}$ tienen sobre la variable dependiente (Enciso, 2014). Se estimaron diversos modelos empleando diferentes combinaciones de variables independientes hasta obtener el modelo que cumpliera los supuestos del modelo de regresión lineal relacionados con multicolinealidad, heterocedasticidad y autocorrelación, así como una adecuada bondad de ajuste con un nivel de confianza de $95 \%$ con el fin de obtener parámetros que pudieran explicar lo más cercano a la realidad los efectos entre variables (Gujarati y Porter, 2010).

\section{Resultados y discusión}

\section{Características de la región}

La región mixteca en el estado de Oaxaca es eminentemente rural, INEGI (2010) reporta que 97\% de los municipios tenían menos de 15000 habitantes, de acuerdo con Gutiérrez y González (2001) las poblaciones rurales se caracterizan por presentar alto porcentaje de analfabetismo, primaria 
como escolaridad predominante, baja disponibilidad de servicios públicos (alumbrado público, agua, transporte, drenaje, servicios de salud) además predomina la agricultura como actividad económica, características de la mayoría de las comunidades oaxaqueñas. En el año 2010, la población ocupada en la mixteca rural equivalía a $19 \%$ de la población total y más de la mitad de ésta se dedicaban al sector primario (55\%), seguido de las actividades secundarias (20\%), servicios (16\%), comercio (8\%) y no especificado (1\%) (INEGI, 2010).

Los niveles de ingresos en la región generalmente han sido bajos (Figura 1), el grueso de la población ocupada gana hasta 1 salario mínimo $(\mathrm{sm})$; no obstante, se registró un cambio importante del año 2010 al 2015, donde el porcentaje de población que ganaba hasta $1 \mathrm{sm}$ disminuyó a la mitad, aumentando las personas que ganaban más de 2 sm y entre 1 y 2 sm (INEGI, 2015). Esta situación se relaciona con el proceso de cambio en las principales actividades económicas, donde la actividad agrícola perdió su papel protagónico en la economía regional y donde el trabajo asalariado desplaza a la actividad agrícola, son parte de las grandes transformaciones que actualmente suceden en la población rural (de Grammont, 2009).

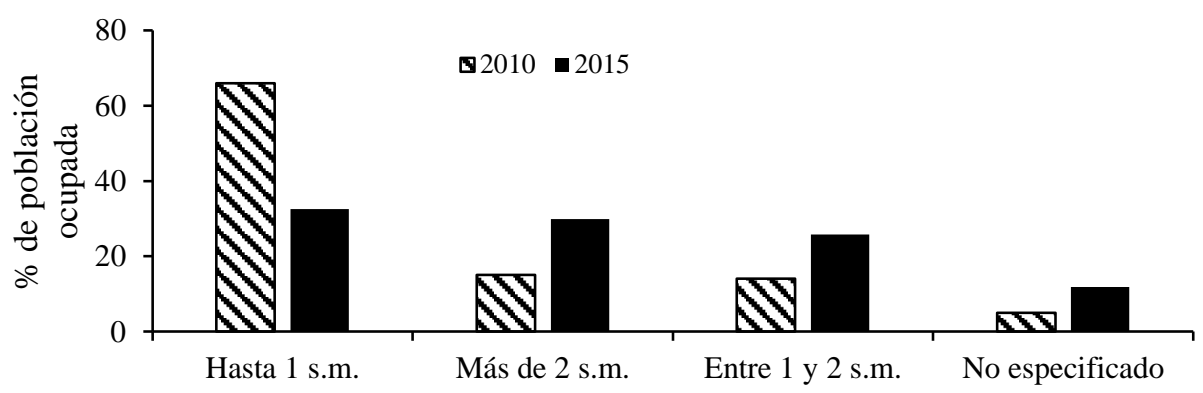

Figura 1. Nivel de ingreso en la región mixteca (INEGI, 2010; 2015).

A pesar de que en los últimos años se ha presentado un relativo aumento de los ingresos, sigue persistiendo la condición de pobreza en la región, para el año 2010; 36\% de la población sufría pobreza alimentaria, $50 \%$ pobreza de capacidades y $70 \%$ pobreza de capacidades de acuerdo con la clasificación de pobreza por ingresos del CONEVAL.

La investigación de Ramales et al. (2014) respaldan la información afirmando que, en el año 2008, $62 \%$ de la población oaxaqueña (2.2 millones de personas) se encontraba en pobreza multidimensional, en 2010 el porcentaje aumentó a 67\% (2.5 millones de personas), resaltan también que la región mixteca ocupa la cuarta posición de pobreza estatal.

Aunado a la pobreza, la población de la región presenta bajos niveles de escolaridad, en el año 2010 más de la mitad de la población mayor de 15 años tenía únicamente educación primaria trunca, para el año 2015 el porcentaje de población con nivel primaria disminuyó aumentando la educación secundaria, pero aun así el grueso de la población siguió en el promedio de 5.6 años de estudios.

Por otra parte, la situación productiva relacionada con la agricultura indica que el estado de Oaxaca aporta $1.5 \%$ al producto interno bruto (PIB) nacional, de este $1.5 \%$ el $6 \%$ corresponde al PIB agropecuario, la mixteca aporta sólo $5 \%$ del valor de la producción agrícola de la entidad. El SIAP por su parte reporta datos sobre el valor de la producción agrícola a nivel Distritos de Desarrollo 
Rural (DDR) de 2003 a 2019, analizando la evolución del valor de la producción antes y después del año de estudio, se observó que el sector agrícola en la mixteca no ha presentado cambios importantes, siendo el cuarto lugar con una tasa de crecimiento anual de $4 \%$ a lo largo de dieciséis años a diferencia de la región oaxaqueña de valles centrales que presentó la mayor tasa de crecimiento con 9\% anual. Si se considera a partir del año de estudio (2007) al año 2019, la tasa de crecimiento anual en la mixteca fue $0.1 \%$ indica que durante este periodo de tiempo es probable que las empresas agrícolas o UP no hayan incrementado su rentabilidad.

En los 151 municipios rurales de la región mixteca, existen 69960 UP, las cuales en su conjunto abarcan 195773 ha del territorio de la mixteca (12.5\% del territorio total). El 80\% de las UP se dedican a las actividades agropecuarias o forestales con el uso de 155672 ha. Las composición y características de las unidades productivas agrícolas por municipio rural son precarias (Cuadro 1).

Cuadro 1. Características por UP de la región mixteca oaxaqueña.

\begin{tabular}{lccccccc}
\hline \multicolumn{1}{c}{ Características } & Media & Mediana & Moda & $\begin{array}{c}\text { Desviación } \\
\text { estándar }\end{array}$ & \multicolumn{3}{c}{ Rango Mínimo Máximo } \\
\hline Superficie (ha) & 2.7 & 2.4 & - & 1.5 & 14.9 & 0.82 & 15.7 \\
Superficie temporal (ha) & 2.5 & 2.4 & - & 1.5 & 15.2 & 0.51 & 15.7 \\
Superficie riego (ha) & 0.16 & 0.04 & 0 & 0.24 & 1.2 & 0 & 1.2 \\
Superficie fertilizada (ha) & 1 & 0.8 & 0 & 1.2 & 12.8 & 0 & 12.8 \\
Superficie con semilla mejorada (ha) & 0.07 & 0.01 & 0 & 0.29 & 3.21 & 0 & 3.21 \\
UP con crédito o seguro & 12 & 4 & 0 & 28 & 192 & 0 & 192 \\
Trabajadores (número) & 3 & 2.6 & 3.3 & 2.2 & 16.7 & 0.06 & 16.8 \\
Trabajadores no remunerados & 1.5 & 1.3 & 1 & 1.05 & 5.9 & 0.04 & 6 \\
Trabajadores remunerados & 1.5 & 0.9 & 0 & 1.6 & 13 & 0 & 13 \\
\hline
\end{tabular}

INEGI (2007).

En promedio existen 350 UP por municipio. Del total de las UP, 96\% son de temporal, porcentaje más alto que el promedio estatal reportado por el Gobierno del Estado de Oaxaca (2016) equivalente al 93\%, de acuerdo con la institución gubernamental este aspecto indica bajo nivel de tecnificación en el proceso agrícola especialmente en cuanto al uso del agua, situación que hace a la agricultura de la región altamente vulnerable a eventos climatológicos. Cabe señalar, que la gran mayoría de los dueños de las UP manejan una pequeña propiedad, pues en promedio cada UP tiene 2.4 ha y ocupa 2.6 trabajadores.

En la región se cosechó una superficie total anual de 112574 ha, lo que en promedio equivale a que cada empresa rural cosechó 1.6 hectáreas, equivalente al 53\% de la superficie promedio de cada unidad productiva. Las empresas rurales de la región tuvieron en total una producción de 125 924 t de cultivos agrícolas, lo que en promedio significa que cada empresa rural produjo $1.8 \mathrm{t}$ para el año 2007, únicamente $29 \%$ de la superficie fue sometida a fertilización, por otra parte, solo $8 \%$ de las empresas rurales utilizaron sistema de riego, además que en la región existe una maquinaria disponible por cada 1892 UP. En total se cultivaron 38 productos; sin embargo, cuatro predominan en superficie, volumen y valor de la producción (Figuras 2, 3 y 4). 


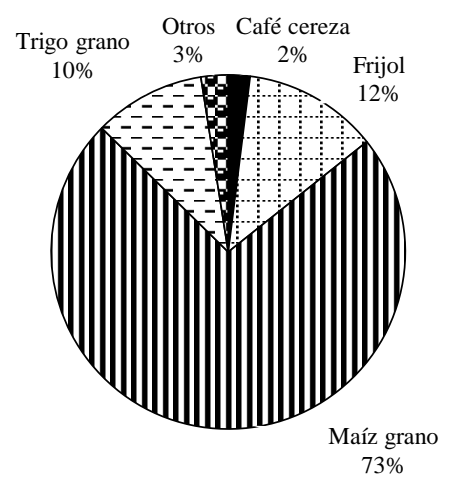

Figura 2. Superficie (ha) cosechada por cultivo (SIAP, 2007).

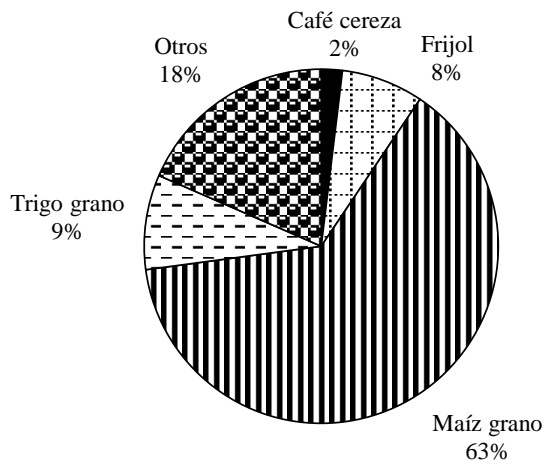

Figura 3. Volumen (t) de producción por cultivo.

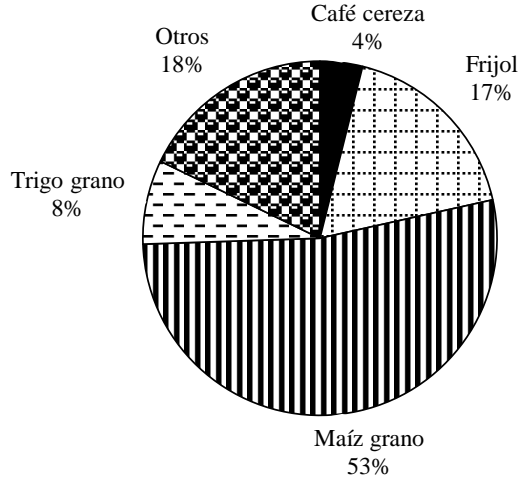

Figura 4. Valor de la producción por cultivo (SIAP, 2007).

Los rendimientos de los cultivos de manera individual, muestran que en la región el cultivo que genera mayor productividad es el café $\left(1.09 \mathrm{t} \mathrm{ha}^{-1}\right)$, seguido por el trigo $\left(0.95 \mathrm{t} \mathrm{ha}^{-1}\right)$, el maíz $(0.94$ $\mathrm{t} \mathrm{ha}^{-1}$ ) y finalmente el frijol $\left(0.55 \mathrm{t} \mathrm{ha}^{-1}\right)$. De acuerdo con Bravo et al. (1992), en la región mixteca, el rendimiento del maíz bajo condiciones de humedad y temperatura adecuadas es de $1.18 \mathrm{t} \mathrm{ha}^{-1}$, el promedio obtenido en este trabajo es inferior, aunque no por mucho ( $\left.0.94 \mathrm{t} \mathrm{ha}^{-1}\right)$. El rendimiento ideal del trigo en la región que indica Bravo $\mathrm{t} \mathrm{ha}^{-1}$, valor muy superior al reportado en esta investigación. 
Por otra parte, Ruiz y Loaeza (2004), reportaron rendimientos de frijol para la región de Valles Centrales de $0.28 \mathrm{t} \mathrm{ha}^{-1}$ bajo el método tradicional y 0.6 con método tecnificado, el rendimiento de frijol obtenido en esta investigación se acerca al cultivo tecnificado realizado en Valles Centrales. De acuerdo a INEGI (1997), el rendimiento promedio del cultivo del café en la entidad es de $1.6 \mathrm{t}$ $\mathrm{ha}^{-1}$, valor ligeramente superior al aquí reportado. Comparando con Montesillo (2016) quien reporta el rendimiento del maíz de temporal nacional de $2.2 \mathrm{t} \mathrm{ha}^{-1}$, el rendimiento del maíz de la mixteca está por debajo en una tonelada, además se encuentra en gran desventaja respecto al maíz de riego que Montesillo reporta de $7.5 \mathrm{t} \mathrm{ha}^{-1} \mathrm{y}$ en el norte del país de $8 \mathrm{t} \mathrm{ha}^{-1}$, incluso los valores obtenidos son inferiores a los que reporta para la zona sur del país $2.8 \mathrm{t} \mathrm{ha}^{-1}$ para maíz de temporal (CNDI, 2008).

En lo concerniente a la mano de obra agrícola, las UP de la región generan trabajo para 175277 personas; sin embargo, $51 \%$ es trabajo no pagado (representa en promedio 1.15 empleos remunerados y 1.30 empleos no remunerados por UP). Debido a que la mayor parte de los municipios de la región emplean mano de obra familiar no remunerada, la agricultura de la región se considera de tipo familiar. Yúñez et al. (2013) definen a la agricultura familiar como aquella unidad de producción agropecuaria o forestal que usa más de $50 \%$ de mano de obra familiar respecto al total de la fuerza de trabajo que emplea. En este sentido $61 \%$ de los municipios estudiados practican agricultura familiar.

Debido a las condiciones de pobreza por ingresos de los campesinos, la producción de autoconsumo cumple un papel fundamental para asegurar la alimentación de las familias, es así que predominan las UP que destinan su producción al consumo familiar y consumo del ganado el cual es una forma de autoconsumo (Cuadro 2). El porcentaje de autoconsumo (consumo familiar más consumo ganadero) se encuentra por arriba del promedio nacional de unidades económicas rurales $(73 \%)$ reportado por Gómez (2016). La información coincide con los estudios de Bolaños (1995) que indican la predominancia de la producción de subsistencia en la región, lo que provoca la minimización del aporte o del valor de la fuerza de trabajo (dado que es mano de obra no remunerada).

Cuadro 2. Destino de la producción de las UP rurales en la Mixteca 2007.

\begin{tabular}{ccc}
\hline Destino de la producción & Núm. de UP & Participación en el número UP (\%) \\
\hline Consumo familiar & 47893 & 70 \\
Consumo ganadero & 7606 & 11 \\
Mercantil & 12605 & 19 \\
Exportación & 10 & 0.01 \\
\hline
\end{tabular}

Base de datos del INEGI (2007).

La caracterización general de la región indica que las empresas rurales se encuentran en un contexto productivo y económico de baja productividad y baja inversión, predomina la pequeña parcela donde se realizan procesos productivos sin la tecnología mecánica mínima necesaria para incrementar la producción, y prácticamente no se hace uso de fertilizantes, esto genera que en promedio la producción por empresa rural sea baja, solo 1.8 t para el año 2007, equivalente a producir aproximadamente $150 \mathrm{~kg}$ por mes o bien, $735 \mathrm{~kg}$ anuales por persona. 


\section{Rentabilidad de las empresas agrícolas rurales}

Para la obtención de los cálculos de rentabilidad se consideraron 144 municipios, eliminando siete debido a incongruencia en su información reportada en el censo (cero trabajadores). Empleando la información de costos reportados por SAGARPA, SENASICA e INIFAP (2015) para los cultivos de la región se lograron obtener estimaciones de rentabilidad agrícola por municipio, por empresa rural y por superficie considerando un escenario de uso mínimo de insumos: Costo de mano de obra: $\$ 77.00$ diarios (sm, vigente en el año 2007).

Los resultados muestran que el rango de rentabilidad en el cual se encuentran las UP va de los \$19000 a $\$ 13$ 000, existiendo casos atípicos (\$-24 733 y \$52 162). Bajo este escenario, únicamente $44 \%$ de las UP generan rentabilidades positivas, pero en general muy bajas (Cuadro 3 ).

Cuadro 3. Rentabilidades de las empresas agrícolas (pesos) por municipio (año 2007).

\begin{tabular}{ccc}
\hline Tipo de UP $^{1}$ & Municipios & Rentabilidad $^{1}$ \\
\hline Consolidada $^{1}$ & $5(3 \%)$ & $>\$ 36150<\$ 139200$ \\
Transición $^{1}$ & $5(3 \%)$ & $\$ 17354$ a $\$ 36150$ \\
Subsistencia $^{1}$ & $54(38 \%)$ & $\$ 0$ a $\$ 17353$ \\
Bajo potencial $^{1}$ & $80(56 \%)$ & $\$-1$ a $\$-24733$ \\
\hline
\end{tabular}

${ }^{1}=$ categorización de acuerdo con SAGARPA (2012).

SAGARPA (2012) realizó una categorización de las unidades productivas de agricultura familiar con potencial productivo considerando su nivel de integración al mercado e indicadores de ingreso bruto o rentabilidad, basándose en las particularidades de dicha categorización: agricultura familiar de subsistencia (AFS), agricultura familiar en transición (AFT) y agricultura familiar consolidada (AFC), se caracterizaron las UP de la mixteca (Cuadro 3). En suma, menos de la mitad de las UP estudiadas se consideran con potencial productivo (44\%) de estas, el mayor porcentaje se consideran AFS que están orientadas exclusivamente al autoconsumo con ingresos insuficientes para garantizar un nivel de vida apropiado recurriendo a otras fuentes de ingresos.

Las UP de AFT y AFC, las cuales tienen el mayor potencial de incrementar la producción y acceder a mercados se localizan, en orden descendente, en los siguientes municipios: San Andrés Sinaxtla, Santiago Tillo, Santa María Chachoápam, San Juan Yucuita, San Juan Sayultepec, San Francisco Chindúa, San Miguel Tecomatlán, Santiago Noyoó, Santo Domingo Yodohino y Santo Domingo Yanhuitlán.

Por otro lado, analizando la rentabilidad a nivel municipal, la actividad agrícola de más de la mitad de los municipios obtiene rentabilidades negativas (Cuadro 4). La mayor parte de los municipios se encuentran en el rango de rentabilidad de menos cuatro millones de pesos a cuatro millones de pesos. Existen municipios que se consideran casos atípicos puesto que sus valores no están dentro de este rango.

Los siete municipios donde la agricultura es más rentable corresponden a Magdalena Jaltepec, Santa María Yucuhiti, Santiago Noyoó, Santa María Chachoápam, San Juan Sayultepec, San Andrés Sinaxtla y Santiago Tillo. 
Cuadro 4. Rentabilidades de la agricultura municipal (pesos) (año 2007).

\begin{tabular}{ccc}
\hline & Municipios & Rentabilidad \\
\hline Categoría I & $7(5 \%)$ & $\$ 6000000$ a 13000000 \\
Categoría II & $57(39 \%)$ & $\$ 1$ a $\$ 5999999$ \\
Categoría III & $80(56 \%)$ & $\$ 0$ a $\$-37000000$ \\
\hline
\end{tabular}

A un nivel más pequeño, las rentabilidades por superficie (ha) van de \$-9 000 por ha hasta $\$ 6500$ por ha, presentándose en casos atípicos valores negativos de hasta \$-20 000 por ha (Cuadro 5).

Cuadro 5. Rentabilidades de la agricultura por hectárea (pesos) (año 2007).

\begin{tabular}{ccc}
\hline & Municipios & Rentabilidad \\
\hline Categoría I & $14(10 \%)$ & $\$ 3000$ a $\$ 7000$ \\
Categoría II & $50(35 \%)$ & $\$ 1$ a $\$ 2999$ \\
Categoría III & $80(55 \%)$ & $\$ 0$ a $\$-20000$ \\
\hline
\end{tabular}

La rentabilidad por hectárea considera los cuatro cultivos en su conjunto; sin embargo, por predominancia en valor de producción, estos resultados explican en mayor parte al cultivo del maíz. El 45\% de los municipios tienen UP que generan ganancias positivas por unidad de superficie. Los valores obtenidos están dentro de los parámetros reportados en otras investigaciones sobre producción rural, por ejemplo, Cruz (2018) encontró ganancias de \$2 012 ha $^{-1}$ para cultivos de maíz en la Sierra Sur de Oaxaca, Ixtla y Santiago (2015) reportaron rentabilidad promedio negativa para el maíz (\$-1 789) en los distritos de Oaxaca.

En el caso de la mixteca existe heterogeneidad en las rentabilidades entre municipios. Los diez municipios donde se localizan las rentabilidades por hectárea más altas son los siguientes: Santa María Chachoápam, San Juan Sayultepec, Santiago Tillo, Santa María Yucuhiti, San Miguel Tecomatlán, San Francisco Chindúa, San Juan Yucuita, Santiago Noyoó, San Andrés Sinaxtla, y Santo Domingo Yodohino.

\section{Factores que influyen en la rentabilidad agrícola}

Mediante la metodología de MCO con corrección de heterocedasticidad se estimaron y evaluaron diversos modelos que intentan explicar cuáles son las variables que influyen de manera significativa sobre la rentabilidad de las empresas rurales en la mixteca, de los modelos estimados, se seleccionó aquel que arrojó la mayor bondad de ajuste, que presentó coeficientes significativos para las variables independientes a un nivel de confianza de $95 \%$ respecto a la variable dependiente, que no mostró colinealidad y que no presentó heterocedasticidad (Cuadro 6, 7,8) el modelo elegido fue el siguiente: Rentabilidad $=-3404.79+19.09$ prod +48.67 supseme-13.67supm-13.71 upautoc -19.26agub-52.38ininsuf-3.32supcom+1620.76supup-9.35up.

Donde: rentabilidad: rentabilidad de las empresas rurales por municipio (pesos); prod: producción agrícola de café, frijol, maíz y trigo (t); supseme: superficie cultivada con semillas mejoradas (ha), supm: superficie cosechada de maíz (ha); upautoc: número de unidades productivas que destinan su producción al autoconsumo; agub: número de unidades productivas con ingresos derivados de 
apoyos gubernamentales; ininsuf: número de unidades productivas con insuficiente infraestructura para la producción (maquinaria, fertilizantes, sistema de riego); supcomun: superficie comunal (ha); supup: superficie por unidad productiva (ha) y up: número de unidades productivas.

Cuadro 6. Modelo 1. MCO con corrección de heterocedasticidad. Variable dependiente: rentabilidad.

\begin{tabular}{cccccc}
\hline & Coeficiente & Desv. típica & Estadístico t & valor $p$ & \\
\hline Const & -3404.79 & 1715.32 & -1.985 & 0.0492 & $* * *$ \\
Prod & 19.0974 & 2.18438 & 8.743 & $<0.0001$ & $* * *$ \\
Supseme & 48.677 & 8.75518 & 5.56 & $<0.0001$ & $* * *$ \\
Supm & -13.6712 & 2.69384 & -5.075 & $<0.0001$ & $* * *$ \\
Upautoc & -13.7139 & 9.57416 & -1.432 & 0.1544 & \\
Agub & -19.2687 & 10.0171 & -1.924 & 0.0565 & $*$ \\
Ininsuf & -52.3869 & 22.0425 & -2.377 & 0.0189 & $* *$ \\
Supcomun & -3.32935 & 0.722753 & -4.606 & $<0.0001$ & $* * *$ \\
Supup & 1620.76 & 551.408 & 2.939 & 0.0039 & $* * *$ \\
Up & -9.35254 & 2.59588 & -3.603 & 0.0004 & $* * *$ \\
\hline
\end{tabular}

Cuadro 7. Estadísticos basados en los datos ponderados.

\begin{tabular}{cccc}
\hline Suma de cuad. residuos & 584.0454 & DT de la regresión & 2.087714 \\
\hline R-cuadrado & 0.909863 & R-cuadrado corregido & 0.903809 \\
F $(9,134)$ & 150.2912 & Valor p (de F) & $1.63 \mathrm{e}-65$ \\
Log-verosimilitud & -305.1391 & Criterio de Akaike & 630.2781 \\
Criterio de Schwarz & 659.9762 & Crit. de Hannan-Quinn & 642.3458 \\
\hline
\end{tabular}

Cuadro 8. Estadísticos basados en los datos originales.

\begin{tabular}{cccc}
\hline Media de la vble. dep. & -40.36425 & DT de la vble. dep. & 12279.88 \\
\hline Suma de cuad. residuos & $1.11 \mathrm{e}+10$ & DT de la regresión & 9086.141 \\
\hline
\end{tabular}

Por cada unidad productiva que no cuente con la infraestructura adecuada para la producción la rentabilidad disminuye $\$ 52.38$. Por cada hectárea de tierra que pertenezca al régimen de tenencia comunal la rentabilidad disminuye \$3.32. Por cada hectárea que conforme la unidad productiva la rentabilidad aumenta $\$ 1$ 620.76. Al incrementarse una unidad productiva en los municipios la rentabilidad disminuye $\$ 9.35$.

\section{Conclusiones}

Las empresas rurales agrícolas son pequeñas en superficie, no disponen de suficiente tecnología y hacen escaso uso de fertilizantes. La rentabilidad de UP osciló en el rango de -19 000 a 13000 pesos, la rentabilidad por municipio fue máximo cuatro millones y la rentabilidad por hectárea fue de -9000 a 6500 pesos. 
Los resultados obtenidos aceptan la hipótesis planteada en la parte que las empresas rurales tienen rentabilidades menores de $\$ 15000$, pero no se acepta en la parte de los factores que influyen a la rentabilidad son mano de obra, fertilización a excepción de la superficie total que, si influye sobre la rentabilidad; es decir, influyen en su mayoría factores relacionados con la tierra, el autoconsumo $\mathrm{y}$ factores externos como los programas gubernamentales.

Se recomienda formar asociaciones de productores que permitan compartir objetivos de producción, incrementar rendimientos y diversificar cultivos, con el fin que el volumen de excedentes pueda competir en los mercados locales o regionales y generar ingresos económicos que incentiven a los productores a continuar con la práctica agrícola como alternativa de trabajo y a invertir en capital que potencialice su proceso de producción. Si bien estas recomendaciones están dirigidas a todos los municipios, los considerados consolidados y en transición tienen las mejores condiciones para crecer en el corto plazo.

\section{Literatura citada}

Bolaños, H. H. 1995. Poverty land degradation, migration and agricultural development: the dynamics of socio-economic transformation in la Mixteca Oaxaqueña, México. Tesis de maestría en Desarrollo Rural. Inglaterra. Universidad de East Anglia. 647-648 pp.

Bravo, E. M.; Van Nieuwkoop, M.; Contreras, J. R.; Jiménez, J. L. y Morales, G. M. 1992. El potencial de la labranza de conservación en la Mixteca Oaxaqueña. INIFAP-CIMMYT. México. $50 \mathrm{p}$.

de Grammont, H. 2009. La desagrarización del campo mexicano. Convergencia. Rev. de Cienc. Soc. 50(16): $13-55$.

CNDI. 2008. Comisión Nacional para el Desarrollo de los Pueblos Indígenas. Condiciones socioeconómicas y demográficas de la población indígena. Región Sur. Tomo 1. Oaxaca. CNDI y PNUD. $200 \mathrm{p}$.

CONEVAL. 2019. Consejo Nacional de Evaluación de la Política de Desarrollo Social. Resultados de pobreza en México 2018 a nivel nacional y por entidades federativas. https://www.coneval.org.mx/Medicion/MP/Paginas/Pobreza-2018.aspx. Octubre de 2019.

CDRSSA. Centro de Estudios para el Desarrollo Rural Sustentable y la Soberanía Alimentaria. 2019. Reporte resultados de la encuesta nacional agropecuaria 2017. Cámara de Diputados LXIV Legislatura. http://www.cedrssa.gob.mx/files/b/13/37ResultadosENA2017.pdf.

Cruz, R. I. 2018. Adopción y rentabilidad de la producción de maíz bajo agricultura de conservación en la región Sierra Sur del Estado de Oaxaca, 2015. Tesis de Ingeniero en Economía Agrícola-Universidad Autónoma Chapingo. Texcoco, México. 89-91 pp.

Enciso, N. R. M. 2014. Evaluación de los factores que influyen en la rentabilidad de la palma aceitera en la región Ucayali. Tesis de Maestro en Ciencias Económicas. Universidad Nacional Agraria de la Selva. Perú. 41-42 pp.

Espinosa, M. E.; García, C. J. L.; Jiménez, V. M. A.; Martínez, S. T.; Pimentel, E. J. L. y Sánchez, E. J. 2013. Caracterización de micro y pequeñas empresas agropecuarias de la región Altos de Chiapas. Rev. Mex. Cienc. Agríc. 6(4):955-962.

Gobierno del Estado de Oaxaca. 2016. Plan estratégico sectorial agropecuario forestal y pesquero. subsector agrícola. Gobierno del Estado de Oaxaca. México. 47 p.

Gómez, O. L. 2016. Evolución del empleo y de la productividad en el sector agropecuario en México. Macroeconomía del desarrollo. CEPAL y FIDA. Chile. 59 p. 
Gujarati, D. N. y Porter, D. C. 2010. Econometría. $5^{\text {ta. }}$ (Ed.). Mc Graw Hill. México, DF. 921 p.

Gutiérrez, M. M. T. y González, S. J. 2001. Propuesta para fijar diez mil habitantes como límite de una localidad urbana. Investigaciones Geográficas. Boletín del Instituto de GeografíaUniversidad Nacional Autónoma de México (UNAM). 44:103-118.

INEGI. 1997. Instituto Nacional de Estadística y Geografía. El maíz en el estado de Oaxaca. INEGI. México. DF. 65 p.

INEGI. 2007. Instituto Nacional de Estadística y Geografía. Censo Nacional Agrícola, Ganadero y Forestal 2007. https://www.inegi.org.mx/programas/cagf/2007/default.html\#Tabulados.

INEGI. 2010. Instituto Nacional de Estadística y Geografía. Censo de Población y Vivienda 2010. https://www.inegi.org.mx/programas/ccpv/2010/.

INEGI. 2015. Instituto Nacional de Estadística y Geografía. México en cifras. Oaxaca. https://www.inegi.org.mx/app/areasgeograficas/?ag=20. Agosto, 2020.

Ixtla, P. Y. y Santiago, Z. M. 2016. Análisis de la rentabilidad y eficiencia de la producción de maíz grano de temporal en los principales DDRs de Oaxaca, 2015. Tesis de Ingeniero en Economía Agrícola. México. Universidad Autónoma Chapingo (UNACH). 47-49 pp.

Mallo, C.; Kaplan, R.; Meljem, S. y Jiménez, C. 2000. Conceptos básicos de economía, empresa y contabilidad de costos y de gestión In: contabilidad de costos y estrategia de gestión. Prentice-Hall. España. 746 p.

Molina de Paredes, O. R. 2017. Rentabilidad de la producción agrícola desde la perspectiva de los costos reales: municipios Pueblo Llano y Rangel del estado Mérida. Venezuela. Venezuela. Visión Gerencial Universidad de los Andes. 1(2):217-232.

Montesillo, C. J. L. 2016. Rendimiento por hectárea del maíz grano en México: distritos de riego vs temporal. Economía Informa. 1(398)60-74.

Ortiz, G. J. y Ríos, B. H. 2013. La pobreza en México, un análisis con enfoque multidimensional. Análisis Económico. 69(28):189-218.

Parra, E. E. 2000. El impacto de la microempresa rural en la economía latinoamericana In: programa de apoyo a la microempresa rural de América Latina y el Caribe. Bustamante, W. (Coord.). Fondo Internacional de Desarrollo Agrícola. Colombia. 36 p.

Ramales, O. M. C.; Coronado, A. M. A. y García, J. R. 2014. Pobreza multidimensional y derechos humanos: situación actual en la mixteca oaxaqueña en el contexto de la política económica nacional. Contribuciones a las Ciencias Sociales. https://www.eumed.net/rev/cccss/29/ mixteca.html.

Ruiz, V. J. y Loaeza, R. G. 2004. Validación del método de siembra en surcos alternos para la asociación maíz-frijol en Valles Centrales de Oaxaca. México. Naturaleza y Desarrollo. $1(2): 13-17$.

SAGARPA. 2012. Secretaría de Agricultura, Ganadería, Desarrollo Rural, Pesca y Alimentación. Agricultura familiar con potencial productivo en México. FAO. México. 534 p.

INIFAP. 2015. Instituto Nacional de Investigaciones Forestales, Agrícolas y Pecuarias- Secretaría de Agricultura, Ganadería, Desarrollo Rural, Pesca y Alimentación (SAGARPA)-Servicio Nacional de Sanidad, Inocuidad y Calidad Alimentaria (SENASICA)-Agenda Técnica Agrícola de Oaxaca. SAGARPA. $2^{\mathrm{a}}$ (Ed.). México. 185 p.

Unikel, L.; Ruiz, C. C. y Garza, G. 1976. El desarrollo urbano de México: diagnóstico e implicaciones futuras. Colegio de México. Centro de Estudios Económicos y Demográficos. México, DF. 476 p.

Universidad Tecnológica de la Mixteca. 2017. Diagnóstico Regional Mixteca. Coordinación General del Comité Estatal de Planeación para el Desarrollo de Oaxaca. México. 11 p.

Yúñez, N. A.; Cisneros, Y. A. y Meza, P. P. 2013. Situando a la agricultura familiar en México. Principales características y tipología. Serie documentos de trabajo. Documento 149. Chile. Centro Latinoamericano para el Desarrollo Rural. 45 p. 